\title{
THE COMBINATION OF HMAX AND HOGS IN AN ATTENTION GUIDED FRAMEWORK FOR OBJECT LOCALIZATION
}

\author{
Tobias Brosch and Heiko Neumann \\ Institute of Neural Information Processing, Ulm University, Ulm, Germany \\ \{tobias.brosch, heiko.neumann\}@uni-ulm.de
}

\begin{abstract}
Keywords: Combination of HMAX and HOGs, Attention, Object localization, Performance evaluation.
Abstract: $\quad$ Object detection and localization is a challenging task. Among several approaches, more recently hierarchical methods of feature-based object recognition have been developed and demonstrated high-end performance measures. Inspired by the knowledge about the architecture and function of the primate visual system, the computational HMAX model has been proposed. At the same time robust visual object recognition was proposed using feature distributions, e.g. histograms of oriented gradients (HOGs). Since both models build upon an edge representation of the input image, the question arises, whether one kind of approach might be superior to the other. Introducing a new biologically inspired attention steered processing framework, we demonstrate that the combination of both approaches gains the best results.
\end{abstract}

\section{INTRODUCTION}

Finding objects in images is a key task for a variety of important applications. The visual input stream provides various features among which edges serve as powerful clues for object detection. Exploiting edge maps, hierarchical methods of feature-based object recognition have recently been developed and demonstrated high-end performance measures. Inspired by the known function and architecture of the primate visual system, the computational HMAX model (Serre et al., 2005; Mutch and Lowe, 2008) has been proposed. At the same time robust visual object recognition was proposed using histograms of oriented gradients (HOGs) (Dalal and Triggs, 2005). Since both models build upon an edge representation of the input image, we explore, whether one kind of feature is superior to the other.

- The HMAX mechanism constitutes a hierarchical model applying iteratively mechanisms of feature combination and pooling. It compares small parts of an intermediate representation in a template like fashion to obtain individual features (Mutch and Lowe, 2008).

- In contrast to the template matching applied in the HMAX model, the HOGs describe local distributions of features derived from the input, namely contrasts in the luminance image. These distributions are calculated for regular-subdivision in in- put images and normalized by the distributions in the discretized neighborhood. HOGs thus can be considered as a likelihood of the presence of certain structure and its distribution in the input data.

Though being quite different in their processing nature, both mechanisms provide some scale and position invariance and build upon an initial edge representation of the input scene. We explore whether the two feature types constitute in a similar way to the classification result or if they significantly facilitate each other when used in combination. This exploration is done proposing a generic coarse-tofine framework for object localization utilizing several standard techniques of past research. It suggests a systematic way to combine multiple processing channels which are by no means limited to the features used in this work. To compare it with previous results, evaluation is done on two different datasets consisting of different object types. We demonstrate state of the art performance on a car data set presented in (Agarwal et al., 2004a) and show that it is also suited for different object categories such as pedestrian on a subset of the very challenging Daimler Pedestrian set (Enzweiler and Gavrila, 2009). The results demonstrate that neither feature type alone represents a complete description of the input and that classification significantly benefits from the combination of both types.

The remaining part of this paper is organized as follows: We describe the novel framework of object localization in section 2 , the data sets are presented 
in section 3 , results are shown in section 4 and finally we discuss the results and the connection to previous work in section 5 .

\section{ARCHITECTURE}

The overall architecture is described in two parts:

1. The basic processing framework combining HMAX and HOGs (histogram of oriented gradients) features to end up with an assembly of view tuned units (VTUs) indicating presence of a target object at a given location and scale.

2. An attention guided region of interest (ROI) selector component inspired by the architecture of (Hamker, 2005). It combines coarse-to-fine processing (Schyns and Oliva, 1994) and the idea of cascaded classifiers (Viola and Michael, 2001; Zhu et al., 2006; Heisele et al., 2001) to focus processing on relevant parts of the input scene.

The suggested processing framework is illustrated in Figure 1.

\subsection{Preprocessing}

The processing starts with a two-level hierarchy. In addition to plain edge detection using a Gabor stage, we introduce a normalization step to compensate different illumination conditions of the input image $I$ by a center surround normalization mechanism given by

$$
I_{\text {norm }}=\frac{I-G_{\sigma} * I}{1+I+G_{\sigma} * I},
$$

where $G_{\sigma}$ is a Gaussian with standard deviation $\sigma=2$ and the operator $*$ denotes the spatial convolution operator. The resulting activities are mapped to a range of $[0,1]$. The edges are extracted by the convolution of $I_{\text {norm }}$ with 2D-Gabor-filters of six different orientations to generate pairs of oriented response maps. The Gabor filters are described by

$$
G(x, y)=\frac{1}{2 \pi \sigma^{2}} \exp \left(-\frac{1}{2} \frac{x^{2}+y^{2}}{\sigma^{2}}\right) \cdot\left(\exp \left(2 \pi i f_{x}^{0} x\right)\right),
$$

where $f_{x}^{0}=0.25$ pixels and $\sigma$ is chosen for halfmagnitude overlap of adjacent Gabor-envelopes (rotated for different orientations). Last, the energy response of the complex-valued Gabor-responses (i.e. the absolute value of the complex filter response) forms the $\mathrm{S} 1$ layer activities which define an $(x, y, \theta)$ space that is input for further processing along two parallel streams, namely HMAX and HOGs.

\subsection{Selective Feature Channels}

After the S1 stage, processing splits into two streams composed of the different feature processing approaches, namely HMAX and HOGs respectively. A discussion of their function follows below.

- HMAX: To provide better comparability to the single scale HOGs features (see below), we decided to employ a single scale HMAX scheme in contrast to the multiple scales and max-pooling across scales in (Mutch and Lowe, 2008). For completeness, we briefly summarize the processing steps of their approach which aim to provide a certain amount of position and scale invariance by iterative application of feature combination (simple cells "S") and pooling mechanisms (complex cells "C"): Layer C1 responses are computed using max-filtering on the S1-layer (Gabor-filter responses) of size $3 \times 3$. During training small patches $P$ of size $n \times n \times p_{s}, n \in$ $\{4,8,12,16\}, p_{s}=6$ ( $p_{s}$ is the number of edge orientations) are extracted from this layer, then sparsified, rated and selected by the classifier. During testing, a patch $P$ is compared to each region $X$ of $\mathrm{C} 1$ units using a Gaussian radial basis function to obtain a similarity estimate of each patch:

$$
R(X, P)=\exp \left(-\frac{\|P-X\|^{2}}{2 \alpha \sigma^{2}}\right),
$$

As in (Mutch and Lowe, 2008) the standard deviation $\sigma$ is set to 1 . To compensate for the effect of comparison in the higher dimensional space in the case of $n \in\{8,12,16\}$, the normalization factor $\alpha$ is set to $\alpha=(n / 4)^{2}$. The maximal response of a patch in a small neighborhood of its original position in its training image forms an entry in the $\mathrm{C} 2$ feature vector. Depending on the searched scale of a target object (i.e. search for a near or distant object) the neighborhood is adjusted. We set the neighborhood arbitrarily to $10 \%$ of the estimated object size (given by the dataset) which seems to work well. This results in different $\mathrm{C} 2$ layers (red, green and blue ellipses in Figure 1), each layer representing an $(x, y, f)$ space, where $f$ is the number of patches or, more generally, the number of features.

- HOGs: We chose the basic variant of HOG features using square cells and square blocks adopting the best parameters as described in (Dalal and Triggs, 2005) (for the pedestrian set we adopted parameters from (Enzweiler and Gavrila, 2009)). To allow for better comparison, we chose the same input (the previously described S1 stage) to both 


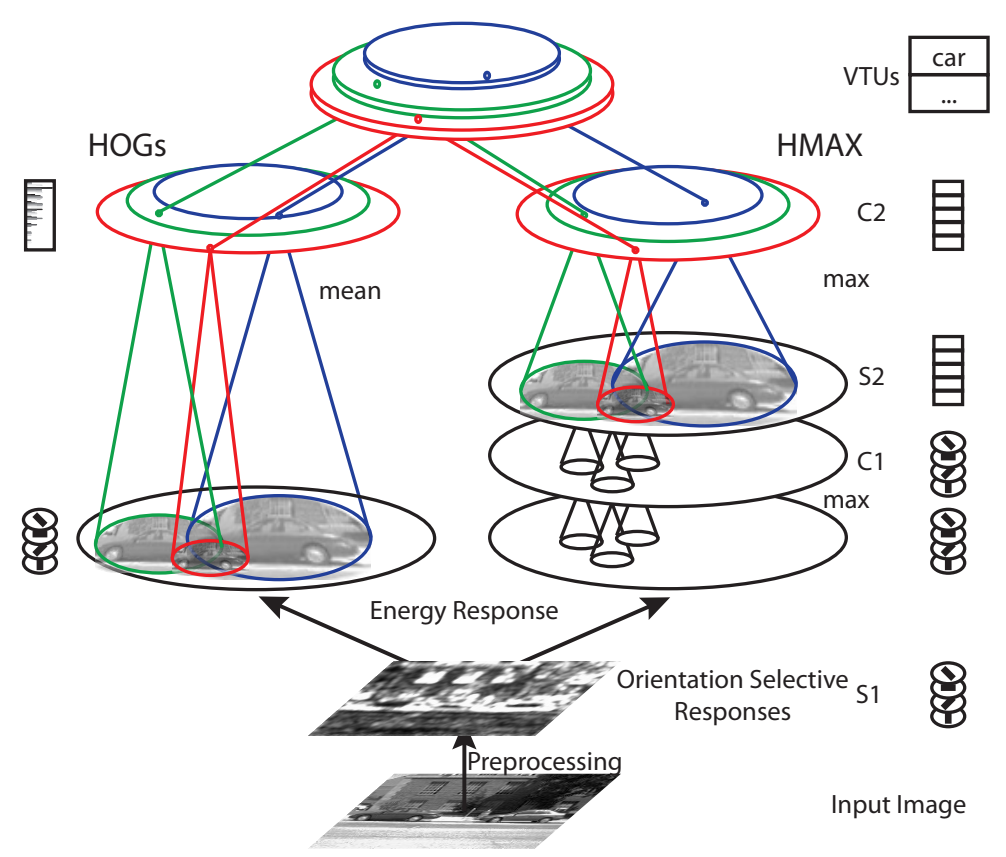

Figure 1: General architecture: The input image is preprocessed and Gabor filtered (layer S1). Subsequent processing results in a feature vector of two different types of features (layer C2, big red ellipses correspond to all locations of small target objects, small blue ellipses to all possible locations of large target objects) at each possible location and scale of a target object. The right side illustrates the HMAX processing, the left side the creation of the HOG features. The final stage of the processing cascade is formed by view tuned units (VTUs). These encode the likelihood of the presence of a target object at a given location and scale (e.g. likelihood of presence of a car). Please note, that for the purpose of better readability, we omitted the component of attentional guidance (please see text section 2.4).

feature types, HMAX and HOGs. Note, however, that according to (Dalal and Triggs, 2005) the common S1 layer (which is chosen to allow comparability to the HMAX features) might not be optimal for HOG features due to the slightly smoothing nature of a Gabor filter. The scene is split into small cells of size $6 \times 6$ pixels across all orientations. For each orientation the mean is taken and normalized across the $3 \times 3$ neighboring cells (so called blocks) using $L 2$ normalization. Similar to adjusting the neighborhood in the HMAX processing stream, we adjusted cell size to end up with the same number of features at each scale (i.e. the layers searching for near and distant objects; red, green and blue circles in Figure 1). In analogy to the HMAX stream, the computations yield a similar $\mathrm{C} 2$ layer structure, wherein each layer represents an $(x, y, f)$ space.

\subsection{Channel Fusion and View-tuned Representations}

The final stage for feature processing is formed by View Tuned Units (VTUs) (Riesenhuber and Poggio, 1999a; Riesenhuber and Poggio, 1999b; Jiang et al.,
2006). A VTU encodes the likelihood of presence of a certain object at its position and scale (e.g. a VTU at the center for large objects specialized for cars). Once the activity of each VTU is known the most likely presence of an object is given by the corresponding object-VTU having maximal activity. To obtain a suitable measure of object presence, we chose an early fusion mechanism (i.e. the combination of our intermediate $\mathrm{C} 2$ feature vectors of each feature type at each position and scale forms the input to a classifier. The activity of a VTU at this location and scale is then given by the output of the classifier). In contrast to a late fusion mechanism, which directly results in VTUs (or another intermediate representation) for each feature type, the early fusion variant employed here has the advantage that no additional fusion mechanism for this second intermediate level is required.

\subsection{Attention Guided Region of Interest Selector Framework}

The extensive search of a large input scene using a strong classifier at several locations leads to an unfavorable computational cost. In order to reduce those costs we apply several concepts and principles of 
computational mechanisms that have been successfully employed in previous work.

- Coarse-to-Fine Processing (CtF). Experimental investigations have shown that early visual processing in humans is dominated by coarse features in contrast to fine grained details which seem to dominate later processing stages (Schyns and Oliva, 1994). From a computational point of view, several studies applied the principle of $\mathrm{CtF}$ processing and reported significant benefits, e.g. (Pedersoli et al., 2010; Amit et al., 2004).

- Cascaded Classifiers. Recent approaches showed that the computational cost of extensive object search can be greatly reduced by applying a cascade of detectors (Viola and Michael, 2001; Zhu et al., 2006; Heisele et al., 2001). Iteration of a process that allows to discard many locations from further processing at an early stage greatly reduces processing time while maintaining a high classification rate.

- Inhibition of Return and Neighborhood Suppression (IOR-Nsupp). Once a suspected or final target position is found the surrounding region can be suppressed, enabling the detection of further candidate locations (IOR) and sparsifying the amount of locations to be searched (Nsupp). This can be formulated in a dynamic framework (Hamker, 2005) as well as in a static context (Agarwal et al., 2004a; Mutch and Lowe, 2008). We apply the neighborhood suppression described in (Agarwal et al., 2004a) with the parameters of (Mutch and Lowe, 2008).

Our attention guided region of interest selector framework combines elementary principles of each of these three mechanisms: We use a simple cascade of two classifiers which already resulted in promising results (this can be extended to support multiple resolution layers as well as multiple classifiers). The first classifier is applied only on the features obtained from the input image that has been downsampled to a quarter of the original resolution ( $\mathrm{CtF}$ processing). To prevent that possible target locations are discarded, the early stage classifier output behavior is biased to a low rejection rate which can be trained and set to a desired false rejection rate (Viola and Michael, 2001; Zhu et al., 2006; Heisele et al., 2001). To evaluate the VTU responses, we apply the IOR-Nsupp mechanism to identify target locations of the next classifier which is applied on the features of the full resolution input (at this level experimental evaluation suggested $60 \%$ of the neighborhood suppression of the final classification stage to facilitate a low rejection rate). After the final classifier is applied, object loca- tion estimates are created under consideration of the IOR-Nsupp mechanism.

\section{TEST SETS}

The evaluation of the proposed model is done on two different data sets. We chose the UIUC car data set (Agarwal et al., 2004a) to compare our model to the results presented in (Mutch and Lowe, 2008). To demonstrate generalizability to a different kind of objects we took a subset of the very challenging Daimler Pedestrian data set (Enzweiler and Gavrila, 2009). Please note, that we chose a one layered architecture of HMAX and that the edge input to HOGs are suboptimal according to (Dalal and Triggs, 2005) in order to allow direct comparability between the two feature types. Consequently the performance of each feature type might suffer a bit compared to the optimal implementation.

\subsection{UIUC Car Data Set}

Figure 2 shows some sample images of the UIUC car data set (Agarwal et al., 2004a). It contains images of side views of cars for use in evaluating object detection algorithms. It comprises 1,050 training images, a single-scale test sequence and 108 multi-scale test images containing 139 cars at various scales and some evaluation files (Agarwal et al., 2004b). We will concentrate on the multiple scales test sequence since our model achieved almost perfect results on the single scale scenario like reported in (Mutch and Lowe, 2008). The evaluation program of (Agarwal et al., 2004 b) results in three measures, namely recall, precision and F-measure (the harmonic mean of precision and recall).

Table 1: Symbols used in defining performance measurement quantities with their accompanying meanings (cf. (Agarwal et al., 2004a)).

\begin{tabular}{c|c} 
symbol & meaning \\
\hline$T P$ & Number of true positives \\
$F P$ & Number of false positives \\
$F N$ & Number of false negatives \\
$n P$ & Total number of positives in data set \\
& $(n P=T P+F N)$
\end{tabular}

Using the notation of table 1 , these are defined as

$$
\operatorname{Rec}=\frac{T P}{T P+F N}=\frac{T P}{n P},
$$




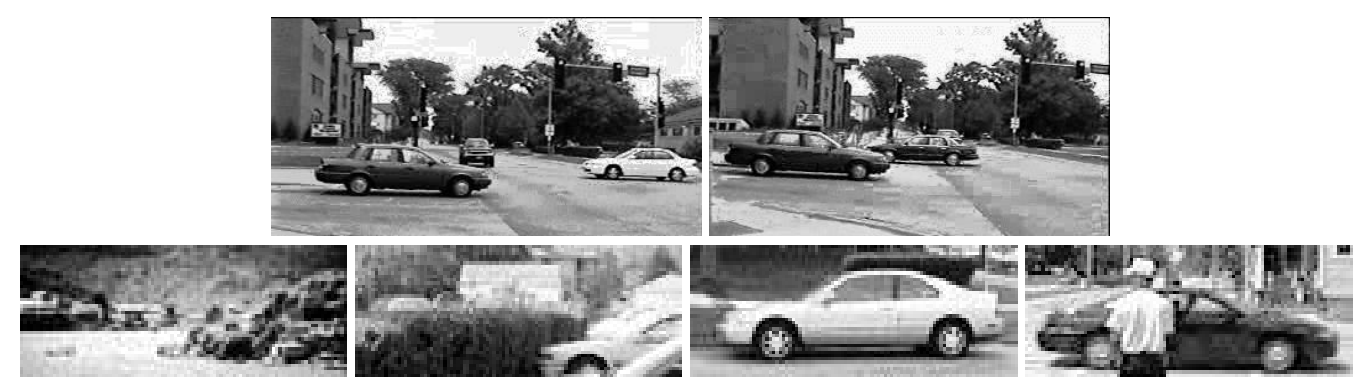

Figure 2: Sample images of the car data set of (Agarwal et al., 2004a). First row: test images containing side-views of cars at multiple scales. Second row: left: negative training images, right: positive training images.

$$
\begin{aligned}
\text { Prec } & =\frac{T P}{T P+F P}, \\
\text { F-measure } & =\frac{2 \cdot \operatorname{Rec} \cdot \text { Prec }}{\text { Rec }+ \text { Prec }} .
\end{aligned}
$$

It is only when both recall and precision have high values that the F-measure is close to one.

\subsection{Daimler Pedestrian Data Set}

Figure 3 visualizes some of the images of the Daimler benchmark (Enzweiler and Gavrila, 2009). The training set contains 15,560 pedestrian samples and 6,744 full images not containing any pedestrians. The test sequence consists of 21,790 images with 56,492 pedestrian labels. It was captured from a vehicle during a 27 min drive through urban traffic at a resolution of $640 \times 480$ pixels. Only pedestrians of a height of at least 72 pixels must be detected. Detection of smaller pedestrians, partially occluded pedestrians, cyclists, etc. are optional and are not counted as detection or false positive. Using the ratio of intersection area and union area of the bounding boxes of a system alarm $a_{i}$ and a ground-truth event $e_{j}$

$$
\Gamma\left(a_{i}, e_{j}\right)=\frac{A\left(a_{i} \cap e_{j}\right)}{A\left(a_{i} \cup e_{j}\right)},
$$

a correct detection is given if $\Gamma\left(a_{i}, e_{j}\right)>0.25$.

Due to computational resource constraints we trained our model on only 5,000 positive and 5,000 negative training images without any bootstrapping and evaluated it on the 1,846 images containing at least one pedestrian of at least 72 pixels height.

\section{RESULTS}

\subsection{Feature Types}

We pose the question whether HMAX or HOGs features encode the same information or whether they provide supplementary information and thus mutually support for each other. This question is quite difficult to answer. From a theoretical point of view, HOGs as well as HMAX features consist of an assembly of nonlinear operations which are hard to compare. Thus we concentrate on an experimental evaluation. Performing initial tests using three different classifiers (AdaBoost (Freund and Schapire, 1997) using a decision tree classifier, Gaussian kernel SVM and linear SVM $^{1}$ we chose a linear SVM classifier which constantly resulted in good classification results at a reasonable training and classification time. We did a systematic evaluation of three different variants of the suggested architecture, the fusion of processing channels (full model, see Figure 1) and ones with only one channel being active. Based on these results, we found that it is the combination of HMAX and HOGs features that results in the best performance compared to either feature type alone (Table 2 shows the results along with comparable previous results. (Lampert et al., 2008) reported a F-measure of $98.6 \%$. However, they used a spatial pyramidal structure that does not compare well to our single scale variant which allows for comparison with HMAX features). Taking into account the standard deviation, the performance gain is significant. To exclude the possibility of a wrong classification bias, we calculated a precision-recall curve which is displayed in Figure 4. It clearly shows that the combination of HMAX and HOGs channels performs best compared to either channel alone.

To assure independence of the evaluated data set, we applied our model to the task of pedestrian detection using a subset of the challenging Daimler Pedestrian set (see section 3.2). We just adjusted the HOG parameters to those given in (Enzweiler and Gavrila, 2009) choosing a scale factor of 1.25 (most similar to their parameter set $S_{6}$ ) and left everything else identi-

\footnotetext{
${ }^{1}$ See (Fradkin and Muchnik, 2006) for more details on support vector machine classifiers; we used the implementation of (Fan et al., 2008).
} 


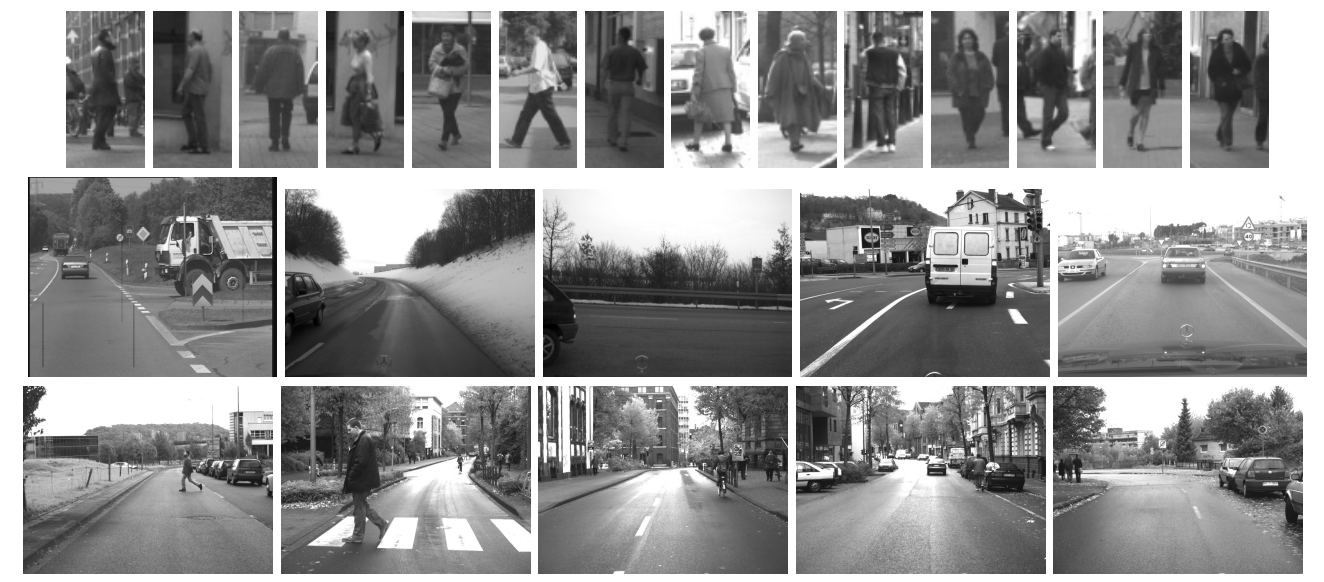

Figure 3: Sample images of the pedestrian data set of (Enzweiler and Gavrila, 2009). First row: positive training images, second row: images containing no pedestrians to generate negative training images and third row: test images.

Table 2: Results on the multiple scales car detection task (at Recall=Precision=F-measure). Scores of our model are the average of 8 independent runs along with standard deviation. Scoring methods were those of (Agarwal et al., 2004a). Note that despite the lower classification rate of our simplified HMAX model compared to (Mutch and Lowe, 2008) and the suboptimal input to the HOG features (to provide comparability), the feature combination of HMAX and HOGs features compensates for it.

\begin{tabular}{l|l} 
Model & Performance \\
\hline Agarwal et al. (Agarwal et al., 2004a) & $39.6 \%$ \\
Fritz et al. (Fritz et al., 2005) & $87.8 \%$ \\
Mutch \& Lowe (Mutch and Lowe, 2008) (sophisticated HMAX) & $90.6 \%$ \\
Our model (only HMAX) & $84.08 \% \pm 1.4 \%$ \\
Our model (only HOGs) & $68.35 \%$ \\
Our model (HMAX\&HOGs) & $\mathbf{9 0 . 8 3 \%} \pm \mathbf{1 . 2 \%}$
\end{tabular}

cal. Due to computational constraints we limited ourselves to a subset of the very large dataset presented in (Enzweiler and Gavrila, 2009) with still considerable extent (see section 3.2 for details, evaluation took multiple weeks of CPU-time). To allow for comparison with the results of (Enzweiler and Gavrila, 2009) we supplemented the precision measure by the false positives per frame measure. The resulting false positives per frame-recall curve is visualized in Figure 5. This confirms that the combination of features gains best performance.

\subsection{Region of Interest Selector Variations}

In section 2.4 we presented a novel region of interest selector framework combining several state of the art techniques. In addition to the examination of HMAX and HOGs features we explored the impact of the different processing stages of our coarse-to-fine architecture. We compared four configurations of our novel model:
1. The entire Coarse to Fine model as described in section 2 .

2. The model using only the Coarse resolution part.

3. A variant of processing the entire image at its Coarse and Fine level (i.e. no selective processing on the high resolution part is done).

4. The model using only the Fine resolution part thus omitting the coarse level and its contribution.

Results are visualized in Figure 6. It shows that each resolution level itself does not perform as well as the model variants using both resolution levels. The two variants using both resolution levels (i.e. the Coarse and Fine and Coarse-to-Fine variants) perform almost identically. This demonstrates that the coarse information is sufficient to arouse attention to the relevant target locations. Even more interesting is the significant benefit of the Coarse-to-Fine architecture. The measured processing time of the high resolution features of the Coarse-to-Fine variant is just about $\mathbf{1 5 \%}$ of the processing time of the Coarse and Fine variant. Of course the processing can be highly parallelized. 


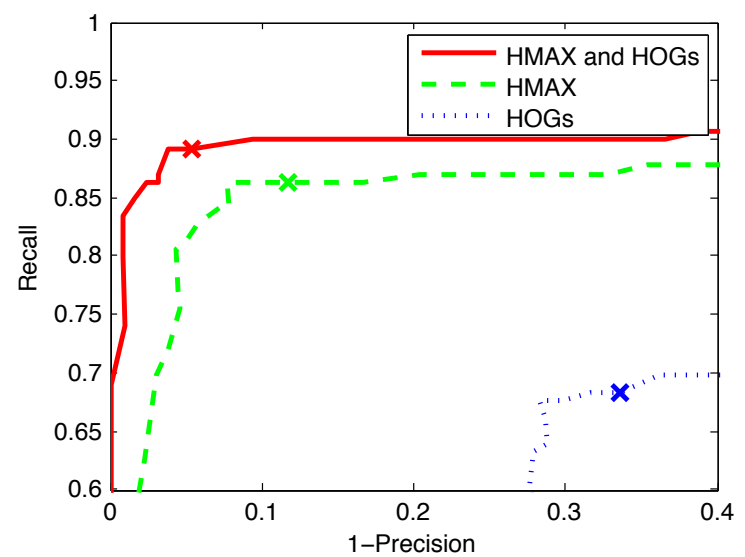

Figure 4: Precision-recall curves of the combination of HMAX and HOG features in comparison to classification results using either feature alone on the UIUC car data set. The "X" marks the system output without any bias. Note, that for the sake of comparison, the input to the HOG features is not optimal.

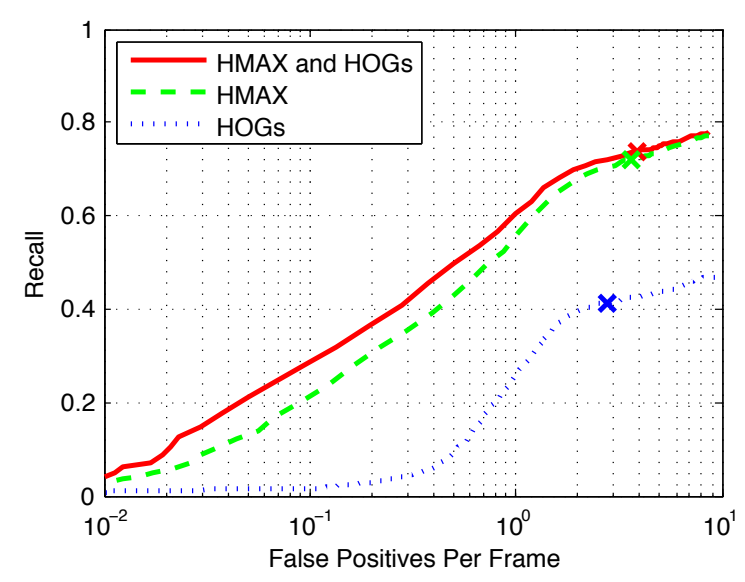

Figure 5: False positives per frame-recall curves of the combination of HMAX and HOG features in comparison to classification results using either feature alone on a subset of the Daimler Pedestrian set. The "X" marks the system output without any bias (note that only a subset was used for training as well as testing; compare to (Enzweiler and Gavrila, 2009) their Figure 6d subset $S_{6}$ ).

However, even if one had unlimited parallel processing resources, the architecture significantly limits the amount of used data. A course resolution image is sufficient to predict target locations with a high precision. This can be very handy in a surveillance scenario there a large region can be watched using a wide angle camera. A controllable camera can then focus on relevant parts of the scene and confirm respectively decline presence of an object at this location.

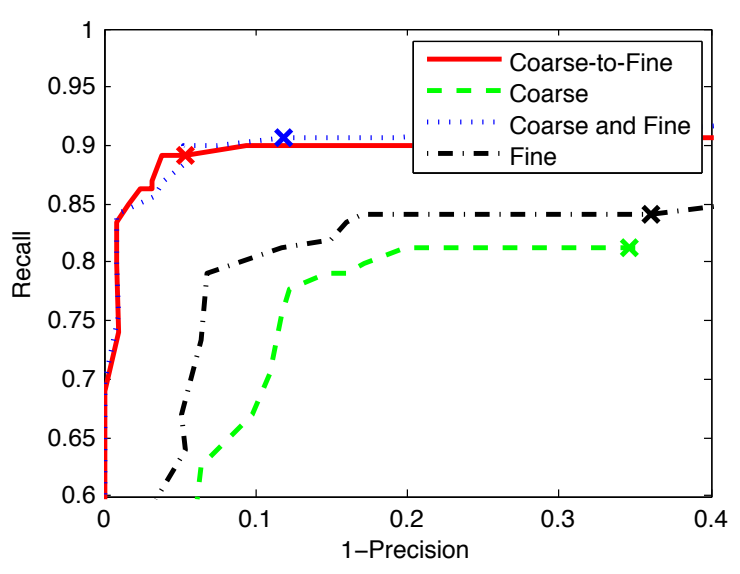

Figure 6: Precision-recall curves of the proposed coarse-tofine architecture in comparison to components of it on the UIUC car data set.

\section{DISCUSSION}

Among several approaches, more recently local feature descriptors based on edge maps demonstrated high-end performance measures. We explored the combination of two commonly used feature descriptors for object detection and localization and our results show that it is worth investigating feature combinations from hierarchical feature processing based on filtering and max-selection (HMAX) as well as feature distributions (HOGs). Our research demonstrates that (though being based on the same input) both feature types facilitate each other and result in improved classification performance in different tasks. Despite using only simple variants of two different processing variants based on the same kind of image features (edges) at only two different spatial resolutions, we showed state of the art performance on the UIUC car data set (Agarwal et al., 2004a). The simplified basic variants of the feature descriptors were chosen to provide comparibility between the two kinds of feature descriptors. Consequently, all results can be further improved by using the complete models as described in the given literature. In addition, we presented a novel general architecture combining elementary principles of previous work in a schematic way that eases extensions in several directions. We demonstrate significant benefits of each of the applied mechanisms. The proposed architecture is meant to serve as a general framework combining different optimization techniques of classification, computational cost reduction as well as combination of different feature types (e.g. coarse-to-fine processing, classifier cascades, neighborhood suppression). It is 
easily extendable by a more advanced classifier cascade (e.g. (Viola and Michael, 2001)) or a cascade of increasingly more complex classifiers (e.g. (Heisele et al., 2001)). These in turn could be combined with efficient subwindow search techniques (e.g. (Lampert et al., 2008; An et al., 2010)). We demonstrated the combination of two feature types. However, in the same way the two feature types have been combined, it can easily be extended by an arbitrary number of additional features. We think that the presented results encourage further investigations in this direction and we will investigate ways of incorporating additional features based on, for example, motion, depth clues, color, etc.

\section{ACKNOWLEDGEMENTS}

We thank the five reviewers for their comments to improve the manuscript. T.B. is supported by a scholarship from the Graduate School of Mathematical Analysis of Evolution, Information, and Complexity at Ulm University. H.N. and T.B. are supported in part by the Transregional Collaborative Research Centre SFB/TRR 62 "Companion-Technology for Cognitive Technical Systems" funded by the German Research Foundation (DFG). We greatly appreciate the computational ressources provided by the (bwGRID, 2011).

\section{REFERENCES}

Agarwal, S., Awan, A., and Roth, D. (2004a). Learning to Detect Objects in Images via a Sparse, Part-Based Representation. TPAMI, 26(11):1475-1490.

Agarwal, S., Awan, A., and Roth, D. (2004b). UIUC Image Database for Car Detection download page. http://12r.cs.uiuc.edu/ cogcomp/Data/Car/. [Online; accessed 27-Mar.-2010].

Amit, Y., Geman, D., and Fan, X. (2004). A Coarse-toFine Strategy for Multiclass Shape Detection. TPAMI, 26(12):1606-21.

An, S., Peursum, P., Liu, W., Venkatesh, S., and Chen, X. (2010). Exploiting Monge Structures in Optimum Subwindow Search. In CVPR.

bwGRID (2011). member of the German D-Grid initiative, funded by the Ministry for Education and Research (Bundesministerium für Bildung und Forschung) and the Ministry for Science, Research and Arts Baden-Wuerttemberg (Ministerium für Wissenschaft, Forschung und Kunst Baden-Württemberg). http://www.bw-grid.de. [Online; accessed 13Apr.-2011].

Dalal, N. and Triggs, B. (2005). Histograms of Oriented Gradients for Human Detection. In CVPR, volume 1, pages 886-893.
Enzweiler, M. and Gavrila, D. M. (2009). Monocular Pedestrian Detection: Survey and Experiments. TPAMI, 31(12):2179-95.

Fan, R., Chang, K.-W., Hsieh, C.-J., Wang, X.-R., and Lin, C.-J. (2008). LIBLINEAR: A Library for Large Linear Classification. JMLR, 9:1871-1874.

Fradkin, D. and Muchnik, I. (2006). Support Vector Machines for Classification. DIMACS Series in Discrete Mathematics and Theoretical Computer Science, 70:13-20.

Freund, Y. and Schapire, R. E. (1997). A DecisionTheoretic Generalization of On-Line Learning and an Application to Boosting. Journal of Computer and System Sciences, 55:119-139.

Fritz, M., Leibe, B., Caputo, B., and Schiele, B. (2005). Integrating Representative and Discriminative Models for Object Category Detection. In ICCV.

Hamker, F. H. (2005). The Emergence of Attention by Population-based Inference and its Role in Distributed Processing and Cognitive Control of Vision. Computer Vision and Image Understanding, 100:64-106.

Heisele, B., Serre, T., Mukherjee, S., and Poggio, T. (2001). Feature Reduction and Hierarchy of Classifiers for Fast Object Detection in Video Images. In CVPR.

Jiang, X., Rosen, E., Zeffiro, T., Vanmeter, J., Blanz, V., and Riesenhuber, M. (2006). Evaluation of a Shape-Based Model of Human Face Discrimination using FMRI and Behavioral Techniques. Neuron, 50(1):159-72.

Lampert, C. H., Blaschko, M. B., and Hofmann, T. (2008). Beyond Sliding Windows: Object Localization by Efficient Subwindow Search. In CVPR, pages 1-8.

Mutch, J. and Lowe, D. G. (2008). Object Class Recognition and Localization using sparse Features with limited Receptive Fields. IJCV, 80(1):45-57.

Pedersoli, M., Gonzàlez, J., Bagdanov, A. D., and Villanueva, J. J. (2010). Recursive Coarse-to-Fine Localization for fast Object Detection. In ECCV, volume 6 .

Riesenhuber, M. and Poggio, T. (1999a). Are Cortical Models Really Bound by the "Binding Problem"? Neuron, 24:87-93.

Riesenhuber, M. and Poggio, T. (1999b). Hierarchical Models of Object Recognition in Cortex. Nature Neuroscience, 2(11):1019-1025.

Schyns, P. G. and Oliva, A. (1994). From Blobs To Boundary Edges: Evidence for Time- and Spatial-ScaleDependent Scene Recognition. Psychological Science, 5(4):195-200.

Serre, T., Wolf, L., and Poggio, T. (2005). Object Recognition with Features inspired by Visual Cortex. In CVPR, pages 994-1000.

Viola, P. and Michael, J. (2001). Rapid Object Detection using a Boosted Cascade of Simple Features. In CVPR.

Zhu, Q., Avidan, S., Yeh, M.-C., and Cheng, K.-T. (2006). Fast Human Detection Using a Cascade of Histograms of Oriented Gradients. In CVPR. 


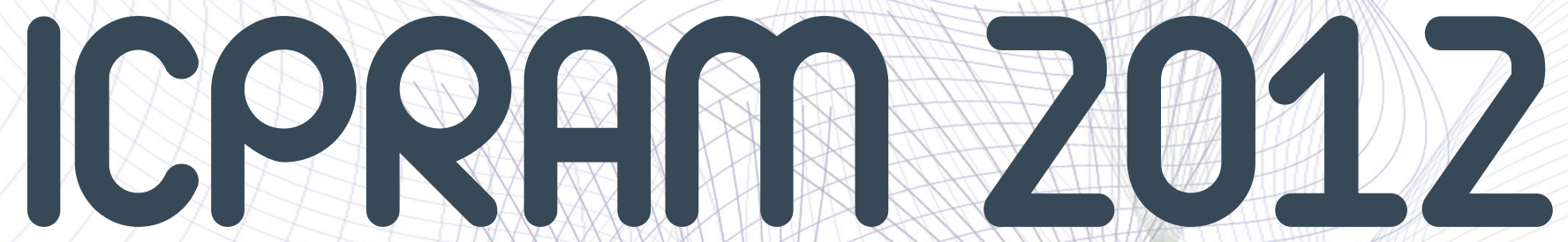

$1^{\text {st }}$ International Conference on Pattern Recognition Applications and Methods

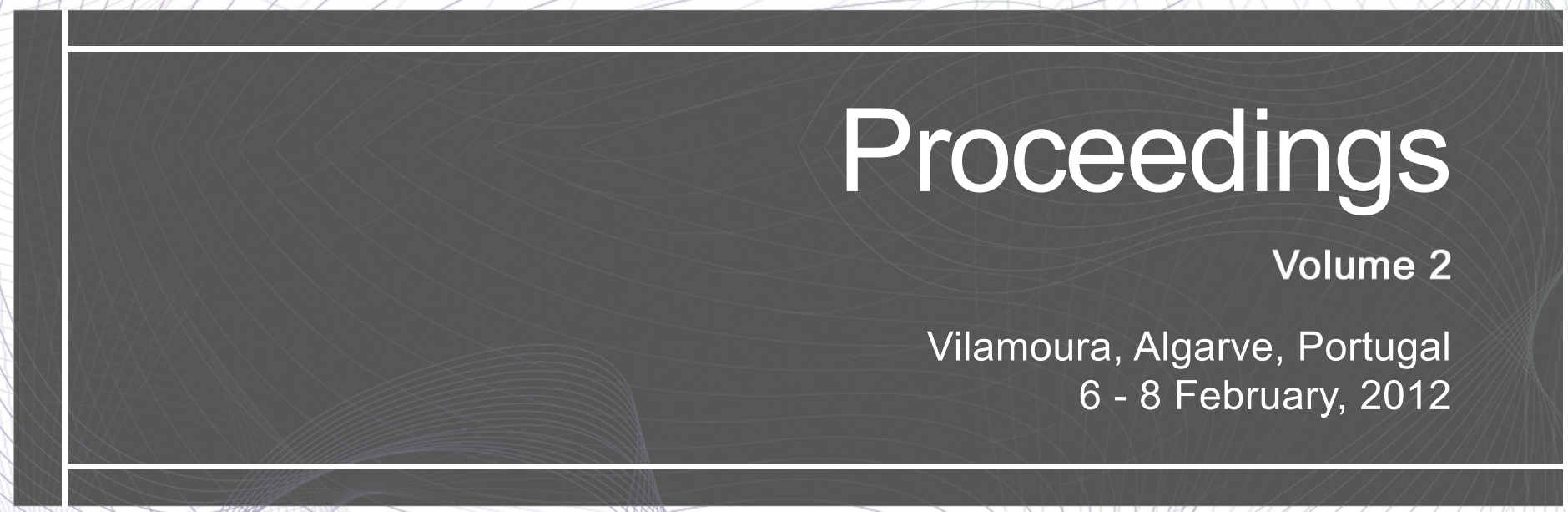

Sponsored by:

\section{IIISTICC}

Technically Co-sponsored by:
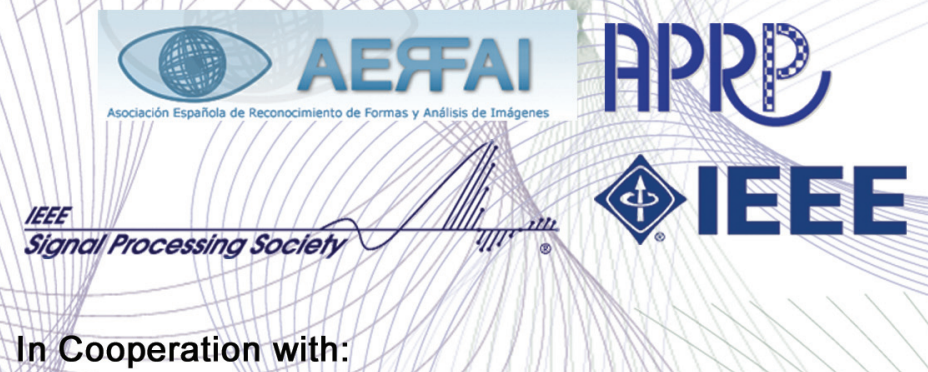


\title{
ICPRAM
}

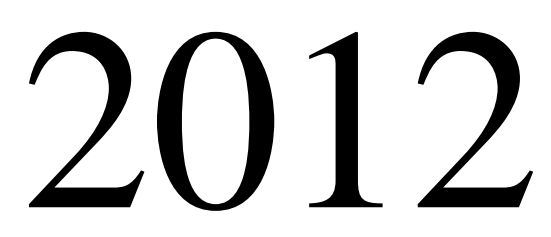

\author{
Proceedings of the \\ 1st International Conference on \\ Pattern Recognition Applications and Methods
}

Volume 2

Vilamoura, Algarve, Portugal

6 - 8 February, 2012

Sponsored by

INSTICC - Institute for Systems and Technologies of Information, Control and Communication

Technically Co-sponsored by

AERFAI - Asociación Española de Reconocimiento de Formas y Análisis de Imágenes

APRP - Associação Portuguesa de Reconhecimento de Padrões IEEE SPS - IEEE Signal Processing Society

IEEE - Institute of Electrical and Electronics Engineers

MLSP - Machine Learning for Signal Processing Technical Committee of IEEE

In Cooperation with

AAAI - Association for the Advancement of Artificial Intelligence

PASCAL 2 - Pattern Analysis, Statistical Modelling and Computational Learning 
Copyright (c) 2012 SciTePress - Science and Technology Publications All rights reserved

Edited by Pedro Latorre Carmona, J. Salvador Sánchez and Ana Fred

\author{
Printed in Portugal
}

ISBN: 978-989-8425-99-7

Depósito Legal: 338347/12

http://www.icpram.org/

icpram.secretariat@insticc.org 